\title{
INTOXICAÇÃO MEDICAMENTOSA POR MOTIVAÇÃO SUICIDA NO BRASIL: UM DESAFIO DA SAÚDE PÚBLICA
}

\section{ARTIGO DE REVISÃO}

SILVA, Junielson Soares da ${ }^{1}$

SILVA, Junielson Soares da. Intoxicação medicamentosa por motivação suicida no Brasil: um desafio da saúde pública. Revista Científica Multidisciplinar Núcleo do Conhecimento. Ano 04, Ed. 09, Vol. 04, pp. 163-174. Setembro de 2019. ISSN: 2448-0959, Link de

acesso: https://www.nucleodoconhecimento.com.br/saude/intoxicacaomedicamentosa

\section{RESUMO}

O presente trabalho, trata-se de uma revisão bibliográfica, e teve como objetivo identificar as principais classes farmacológicas envolvidas em casos de intoxicação por motivação suicida, bem como gênero e faixa etária mais afetada. Utilizou as plataformas de busca Google Scholar e LILACS. Selecionou-se 14 artigos publicados entre 2011 a 2017, que foram analisados e apresentados em tabelas e gráficos. Os resultados apontaram que os antidepressivos e os anticonvulsionantes são as classes de medicamentos mais utilizadas em intoxicação com motivação suicida, sendo que os indivíduos com faixa etária entre 20 a 40 anos de idade e do sexo feminino são os mais afetados. O uso de medicamentos com intenção de autoextermínio é um problema de saúde pública no Brasil, mas poucas pesquisas têm se debruçado no sentido de compreender os tipos farmacológicos mais utilizados, sendo necessário tal identificação para o desenvolvimento de políticas de prevenção e promoção à saúde, principalmente daqueles mais vulneráveis.

\footnotetext{
${ }^{1}$ Mestrado em Genética, Conservação e Biologia Evolutiva, especialista em Educação Ambiental e em Saúde Pública, graduado em Ciências Biológicas.
} 
Palavras-chave: suicídio, medicamentos, intoxicação, faixa etária, gênero.

\section{INTRODUÇÃO}

O suicídio é um problema de saúde pública, tem sido objeto de pesquisa em diversos campos do conhecimento e pelo menos $90 \%$ dos casos estão relacionados a transtorno mental (BOTEGA et al., 2010). O comportamento suicida é decorrente de uma combinação de fatores psicológicos e biológicos, inclusive genéticos, culturais e socioambientais, e inclui pensamentos, planos e tentativa, podendo chegar à consumação (ASSOCIAÇÃO BRASILEIRA DE PSIQUIATRIA-ABP, 2014).

No Brasil a taxa de suicídio chegou a 5,3 por 100 mil habitantes, em 2012, cerca de três mil por dia (BOTEGA et al., 2010; WAISELFISZ, 2014; ZHONG; CHIU; CONWEL, 2016). Os medicamentos, embora elaborados com finalidade profilática, curativa e paliativa, é a segunda substância exógena mais utilizada em casos de intoxicação intencional, podendo inclusive levar a óbito (WORLD HEALTH ORGANIZATIONWHO, 2002; SPILLER; APPANA; BROCK, 2008). Em 2014, o Sistema Nacional de Informações Tóxico-Farmacológicas (Sinitox) da Fiocruz, registrou 7.509 tentativas de suicídio e 41 óbitos com uso de medicamentos (FIOCRUZ, 2014).

Várias pesquisas destacam a relação entre intoxicação medicamentosa e suicídio, fato que já ocorre na maioria dos países desenvolvidos, onde essas substâncias ocupam a primeira posição nos casos de intoxicações intencionais (GUNNELL; EDDLESTON, 2003; CABALLERO-VALLES et al., 2008; VÄRNIK et al., 2009), sendo as mulheres as maiores vítimas (MORAIS et al., 2008; MOREIRA et al., 2015).

Contudo no Brasil, poucos estudos destacam as principais classes farmacológicas utilizadas em casos de intoxicação medicamentosa intencional e não foram encontrados estudos que estimem a taxa de mortalidade por suicídio provocados por cada classe. Sendo assim, o presente estudo teve como objetivo realizar uma revisão bibliográfica sobre as intoxicações medicamentosas por motivação suicida no Brasil, entre janeiro de 2011 a dezembro de 2017 e identificar as principais classes farmacológicas envolvidas, bem como gênero e faixa etária das vítimas. 


\section{METODOLOGIA}

O presente trabalho trata-se de uma revisão da literatura. Este tipo de estudo tem por finalidade responder determinada questão científica, apresentar críticas, identificar pontos positivos e negativos dos artigos analisados, e sugerir novas pesquisas, quando necessário (BAPTISTA; MORAIS; SISTO, 2007).

A busca dos artigos em português, publicados no Brasil, entre janeiro de 2011 a dezembro de 2017, foi feito nas bases de dados Scielo, Google Scholar e LILACS (Literatura Latino-Americana e do Caribe em Ciências de Saúde), utilizando os descritores em português: "medicamentos and/or suicídio", "intoxicação and/or suicídio", "fármaco and/or intoxicação" e "fármaco and/or suicídio"

Como critérios de inclusão dos artigos, utilizou-se: publicações derivadas de estudos realizados no Brasil, em língua portuguesa, que abordassem simultaneamente informações sobre suicídio e classes farmacológicas. Os critérios de exclusão foram os artigos repetidos, de revisão da literatura, relatos de caso, em outros idiomas e fora do recorte temporal.

Foram encontrados cerca de 580 artigos, que após os critérios de inclusão e exclusão, restaram 35, e ao serem analisados na integra, apenas 14 deles se adequaram aos critérios. Sendo assim, os artigos foram analisados minuciosamente e o dados estão apresentados a seguir.

\section{RESULTADOS E DISCUSSÃO}

Nas buscas foram encontrados alguns artigos que destacam os medicamentos em tentativas de autoextermínio, mas não buscaram investigar as classes farmacológicas utilizadas (FEUSER, 2013; OLIVEIRA; SUCHARA, 2014a; MACHADO; SANTOS, 2015; VIEIRA; CAVEIÃO, 2016)· Na análise dos artigos, levou-se em consideração o título, autores da pesquisa, ano de publicação e os resultados da pesquisa, que são o alvo deste estudo (classes farmacológicas mais utilizadas, faixa etária e gênero) sobre o suicídio por medicamentos, como mostra a tabela 01. A maioria dos artigos 
publicados são provenientes de estudos realizados na região Nordeste e Sul do país, com cinco e três, respectivamente, as regiões Centro-Oeste e Sudeste com dois artigos, cada uma, não foi encontrado artigo com estudo na região Norte e dois deles analisaram dados nacionais.

Tabela 01: Caracterização do estudo por autor, ano, título do artigo e resultados.

\begin{tabular}{|c|c|c|c|c|}
\hline Autor/Ano & Título do artigo & $\begin{array}{ll}\text { Local da } \\
\text { coleta dos } \\
\text { dados }\end{array}$ & $\begin{array}{l}\text { Local de } \\
\text { estudo }\end{array}$ & $\begin{array}{l}\text { Classe } \\
\text { farmacológica }\end{array}$ \\
\hline $\begin{array}{l}\text { Souza et } \\
\text { al., } 2011\end{array}$ & $\begin{array}{l}\text { Tentativa de } \\
\text { suicídio } \\
\text { mortalidade por } \\
\text { suicídio em um } \\
\text { município no } \\
\text { interior da Bahia }\end{array}$ & $\begin{array}{l}\text { Corpo de } \\
\text { Bomb., } \\
\text { Datasus e } \\
\text { IML }\end{array}$ & Jequié-BA & $\begin{array}{l}\text { Analgésico, } \\
\text { antipiréticos, } \\
\text { antirreumáticos, } \\
\text { antiepilépticos, } \\
\text { sedativos } \\
\text { psicotrópicos }\end{array}$ \\
\hline $\begin{array}{l}\text { Mota et al., } \\
2012\end{array}$ & $\begin{array}{l}\text { Perfil da } \\
\text { mortalidade por } \\
\text { intoxicação com } \\
\text { medicamentos } \\
\text { no Brasil, 1996- } \\
2005 \text { : retrato de } \\
\text { uma década }\end{array}$ & $\begin{array}{l}\text { SIM/ } \\
\text { Ministério da } \\
\text { Saúde }\end{array}$ & Brasil & $\begin{array}{l}\text { Anticonvulsionantes, } \\
\text { sedativos, } \\
\text { antiparkinsonianos e } \\
\text { psicotrópicos }\end{array}$ \\
\hline $\begin{array}{l}\text { Magalhães } \\
\text { et al., } 2013\end{array}$ & $\begin{array}{l}\text { Caracterização } \\
\text { das intoxicações } \\
\text { medicamentosas } \\
\text { registradas no } \\
\text { Centro de } \\
\text { Informações } \\
\text { Toxicológicas do } \\
\text { Piauí no período } \\
\text { de } 2007 \text { a } 2012\end{array}$ & $\begin{array}{l}\text { Centro de } \\
\text { Informações } \\
\text { Toxicológicas }\end{array}$ & Piauí & $\begin{array}{l}\text { Benzodiazepínicos, } \\
\text { anticonvulsionantes, } \\
\text { antidepressivos e } \\
\text { neurolépticos }\end{array}$ \\
\hline
\end{tabular}




\begin{tabular}{|c|c|c|c|c|}
\hline $\begin{array}{l}\text { Santos, } \\
\text { Legay } \\
\text { Lovisi, } \\
2013\end{array}$ & $\begin{array}{l}\text { Substâncias } \\
\text { tóxicas } \\
\text { tentativas de } \\
\text { suicídios: } \\
\text { considerações } \\
\text { sobre o acesso e } \\
\text { medidas } \\
\text { restritivas }\end{array}$ & $\begin{array}{l}\text { SIM, Inform. } \\
\text { Hospitalares } \\
\text { do SUS (SIH- } \\
\text { SUS) e Min. } \\
\text { Da Saúde }\end{array}$ & Brasil & Psicotrópicos \\
\hline $\begin{array}{l}\text { Santos et } \\
\text { al., } 2013\end{array}$ & $\begin{array}{lr}\text { Suicídio } & \text { e } \\
\text { tentativas } & \text { de } \\
\text { suicídio } & \text { por } \\
\text { intoxicação } & \\
\text { exógena no } & \text { Rio } \\
\text { de Janeiro } & \end{array}$ & $\begin{array}{l}\text { Centro de } \\
\text { Controle de } \\
\text { Intoxicações } \\
\text { de Niterói } \\
\text { (CCln) e } \\
\text { Sinan }\end{array}$ & Niterói - RJ & Psicotrópicos \\
\hline $\begin{array}{l}\text { Oliveira e } \\
\text { Suchara, } \\
2014\end{array}$ & $\begin{array}{l}\text { Intoxicações } \\
\text { medicamentosas } \\
\text { em hospital } \\
\text { público de Barra } \\
\text { do Garças - MT, } \\
\text { no período de } \\
2006 \text { a } 2009\end{array}$ & $\begin{array}{l}\text { SIM e } \\
\text { Hospital } \\
\text { Municipal de } \\
\text { Barra do } \\
\text { Garças -MT }\end{array}$ & $\begin{array}{l}\text { Barra do } \\
\text { Garças - } \\
\text { MT }\end{array}$ & $\begin{array}{l}\text { Anestésicos, } \\
\text { analgésicos, } \\
\text { antiepilépticos, } \\
\text { antiparksonianos, } \\
\text { psicolépticos } \\
\text { psiconalépticos }\end{array}$ \\
\hline $\begin{array}{l}\text { Takahama; } \\
\text { Turini } \\
\text { Giroto, } \\
2014\end{array}$ & $\begin{array}{l}\text { Perfil das } \\
\text { exposições a } \\
\text { medicamentos } \\
\text { por mulheres em } \\
\text { idade } \\
\text { reprodutiva } \\
\text { atendidas por um } \\
\text { Centro de } \\
\text { Informações } \\
\text { Toxicológicas }\end{array}$ & CIT & $\begin{array}{l}\text { Londrina- } \\
\text { PR }\end{array}$ & $\begin{array}{l}\text { Antiepiléptico, } \\
\text { antidepressivos, } \\
\text { ansiolíticos, } \\
\text { analgésicos } \\
\text { antipiréticos, } \\
\text { antipsicóticos }\end{array}$ \\
\hline
\end{tabular}




\begin{tabular}{|c|c|c|c|c|}
\hline $\begin{array}{l}\text { Alexandre } \\
\text { e Martins, } \\
2015\end{array}$ & $\begin{array}{l}\text { Avaliação da } \\
\text { frequência } \\
\text { mortalidade } \\
\text { entre adultos } \\
\text { jovens por } \\
\text { intoxicação } \\
\text { medicamentosa } \\
\text { n a 14ª Regional } \\
\text { de Saúde do } \\
\text { Paraná }\end{array}$ & SINAN & $\begin{array}{l}\text { Paranavaí- } \\
\text { PR }\end{array}$ & $\begin{array}{l}\text { Anticonvulsionantes } \\
\text { e antidepressivos }\end{array}$ \\
\hline $\begin{array}{l}\text { Costa e } \\
\text { Alonzo, } \\
2015\end{array}$ & $\begin{array}{l}\text { Casos } \\
\text { exposições } \\
\text { intoxicações } \\
\text { medicamentos } \\
\text { registrados em } \\
\text { um Centro de } \\
\text { Controle } \\
\text { intoxicações de } \\
\text { interior } \\
\text { Estado de São } \\
\text { Paulo }\end{array}$ & $\begin{array}{l}\text { Centro de } \\
\text { Controle de } \\
\text { intoxicações }\end{array}$ & $\begin{array}{l}\text { Campinas } \\
-S P\end{array}$ & $\begin{array}{l}\text { Benzodiazepínicos e } \\
\text { antidepressivos } \\
\text { tricíclicos } \\
\text { Carbamazepina }\end{array}$ \\
\hline $\begin{array}{l}\text { Moreira et } \\
\text { al., } 2015\end{array}$ & $\begin{array}{lr}\text { Perfil } & \text { de } \\
\text { pacientes } & \\
\text { atendidos } & \text { por } \\
\text { tentativa } & \text { de } \\
\text { suicídio em } & \text { um } \\
\text { centro } & \text { de } \\
\text { assistência } & \\
\text { toxicológica. } & \end{array}$ & $\begin{array}{l}\text { Centro de } \\
\text { Assist. } \\
\text { Toxicológica } \\
\text { de um } \\
\text { hospital de } \\
\text { Fortaleza }\end{array}$ & $\begin{array}{l}\text { Fortaleza- } \\
\text { CE }\end{array}$ & $\begin{array}{l}\text { Anticonvulsionantes, } \\
\text { antidepressivos, } \\
\text { ansiolíticos } \\
\text { antipsicóticos }\end{array}$ \\
\hline $\begin{array}{l}\text { Oliveira et } \\
\text { al., } 2015\end{array}$ & $\begin{array}{ll}\text { Tentativa } & \text { de } \\
\text { suicídio } & \text { por }\end{array}$ & $\begin{array}{l}\text { SINAN } \\
\text { Núcleo }\end{array}$ & Ceará & $\begin{array}{l}\text { Ansiolíticos } \\
\text { antidepressivos }\end{array}$ \\
\hline
\end{tabular}




\begin{tabular}{|c|c|c|c|c|}
\hline & $\begin{array}{l}\text { intoxicação } \\
\text { exógena: } \\
\text { contexto de } \\
\text { notificações } \\
\text { compulsórias. }\end{array}$ & $\begin{array}{l}\text { Hospitalar de } \\
\text { Epidemiologia }\end{array}$ & & \\
\hline $\begin{array}{l}\text { Vieira, } \\
\text { Santana e } \\
\text { Suchara, } \\
2015\end{array}$ & $\begin{array}{l}\text { Caracterização } \\
\text { de Tentativas de } \\
\text { suicídio por } \\
\text { substâncias } \\
\text { exógenas }\end{array}$ & SINAN & $\begin{array}{l}\text { Barra do } \\
\text { Garça } \\
\text { MT }\end{array}$ & $\begin{array}{l}\text { Fármacos do } \\
\text { sistema nervoso, } \\
\text { aparelho digestivo e } \\
\text { metabolismo, } \\
\text { aparelho aparelho } \\
\text { geniturinário, } \\
\text { hormônios sexuais e } \\
\text { do respiratório }\end{array}$ \\
\hline $\begin{array}{l}\text { Brixner et } \\
\text { al., } 2016\end{array}$ & $\begin{array}{l}\text { Incidência das } \\
\text { tentativas de } \\
\text { suicídio por } \\
\text { sobredose } \\
\text { intencional de } \\
\text { medicamentos }\end{array}$ & $\begin{array}{l}\text { Prontuário do } \\
\text { Sistema MV } \\
2000\end{array}$ & $\begin{array}{l}\text { Interior do } \\
\text { RS }\end{array}$ & $\begin{array}{l}\text { Ansiolíticos, } \\
\text { antidepressivos, } \\
\text { antipsicóticos, } \\
\text { anticonvulsionantes, } \\
\text { anti-inflamatórios, } \\
\text { psicoestimulantes, } \\
\text { diuréticos e anti- } \\
\text { hipertensivos. }\end{array}$ \\
\hline $\begin{array}{l}\text { Gondim et } \\
\text { al. } 2017\end{array}$ & $\begin{array}{l}\text { Tentativas de } \\
\text { suicídio por } \\
\text { exposição a } \\
\text { agentes tóxicos } \\
\text { registradas em } \\
\text { um Centro de } \\
\text { Informação e } \\
\text { Assistência } \\
\text { Toxicológica em }\end{array}$ & $\begin{array}{l}\text { Centro de } \\
\text { Informação e } \\
\text { Assistência } \\
\text { Toxicológica } \\
\text { (CEATOX) }\end{array}$ & $\begin{array}{l}\text { Fortaleza- } \\
\text { CE }\end{array}$ & $\begin{array}{l}\text { Antipsicóticos, } \\
\text { antidepressivos, } \\
\text { antiepiléticos } \\
\text { ansiolíticos }\end{array}$ \\
\hline
\end{tabular}




\section{Fortaleza, \\ Ceará, 2013.}

Fonte: Autor.

Houve variação dos artigos publicados período de 2011 a 2017, com o tema "suicídio e classe farmacológica". Os anos com o maior número de publicações foram 2015, com cinco artigos (38,46\%), 2013, com três $(23,07 \%)$ e 2014 , com dois $(15,38 \%)$, nos demais anos apenas um artigo contendo informações requisitadas foi publicado (Figura 1). Baseado na oscilação das publicações no período investigado, não é possível afirmar se houve aumento ou redução, nem mesmo que permaneceu constante número de artigos publicados.

Figura 1: Distribuição dos artigos com a temática "suicídio e classe farmacológica" por ano, no período de 2011 a 2017, no Brasil.

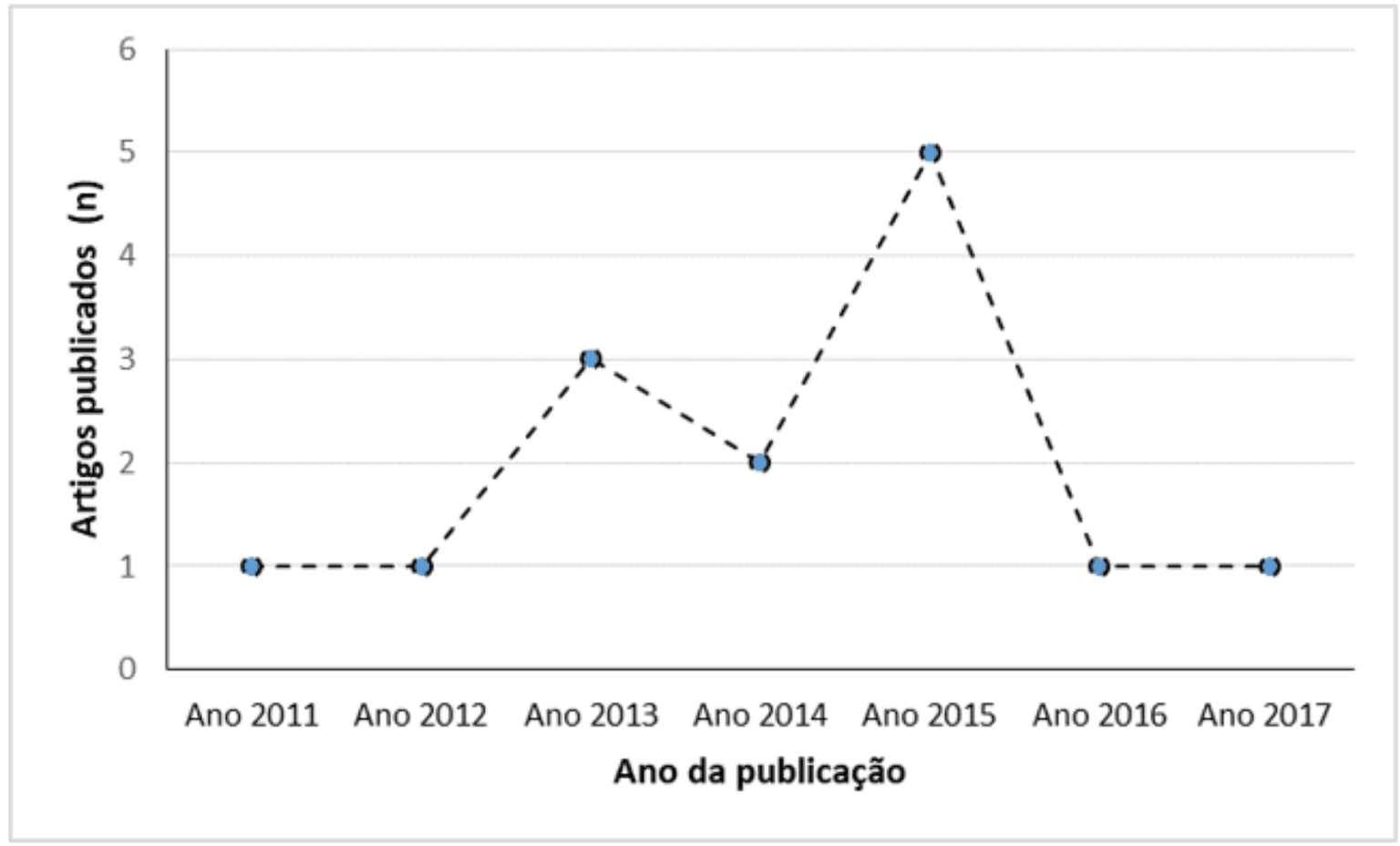

Fonte: Autor.

Os tipos farmacológicos apontados nos artigos como os que mais causaram intoxicações por motivação suicida, foram os antidepressivos, seguido dos RC: 6928

Disponível em: https://www.nucleodoconhecimento.com.br/saude/intoxicacao-medicamentosa 
anticonvulsionantes, ansiolíticos, antipsicóticos e psicotrópicos, respectivamente. Os analgésicos, antipiréticos, antiepilépticos e benzodiazepínicos também foram apontados como os mais utilizados (Figura 2). Outros tipos de medicamentos (antiinflamatórios, antiparkisonianos, sedativos, dentre outros) foram menos verificados quanto ao uso com intenção de autodestruição, mas a variedade de tipos farmacológicos, incluindo aqueles de uso restrito é um fato que preocupa, pois percebe-se que há uma facilidade de acesso aos medicamentos, incluindo aqueles que deveriam ser prescritos apenas por psicólogos ou médicos psiquiatras.

Figura 2: Classes farmacológicas utilizadas em casos de suicídio, citadas nos artigos analisados com publicação entre 2011 a 2017, no Brasil.

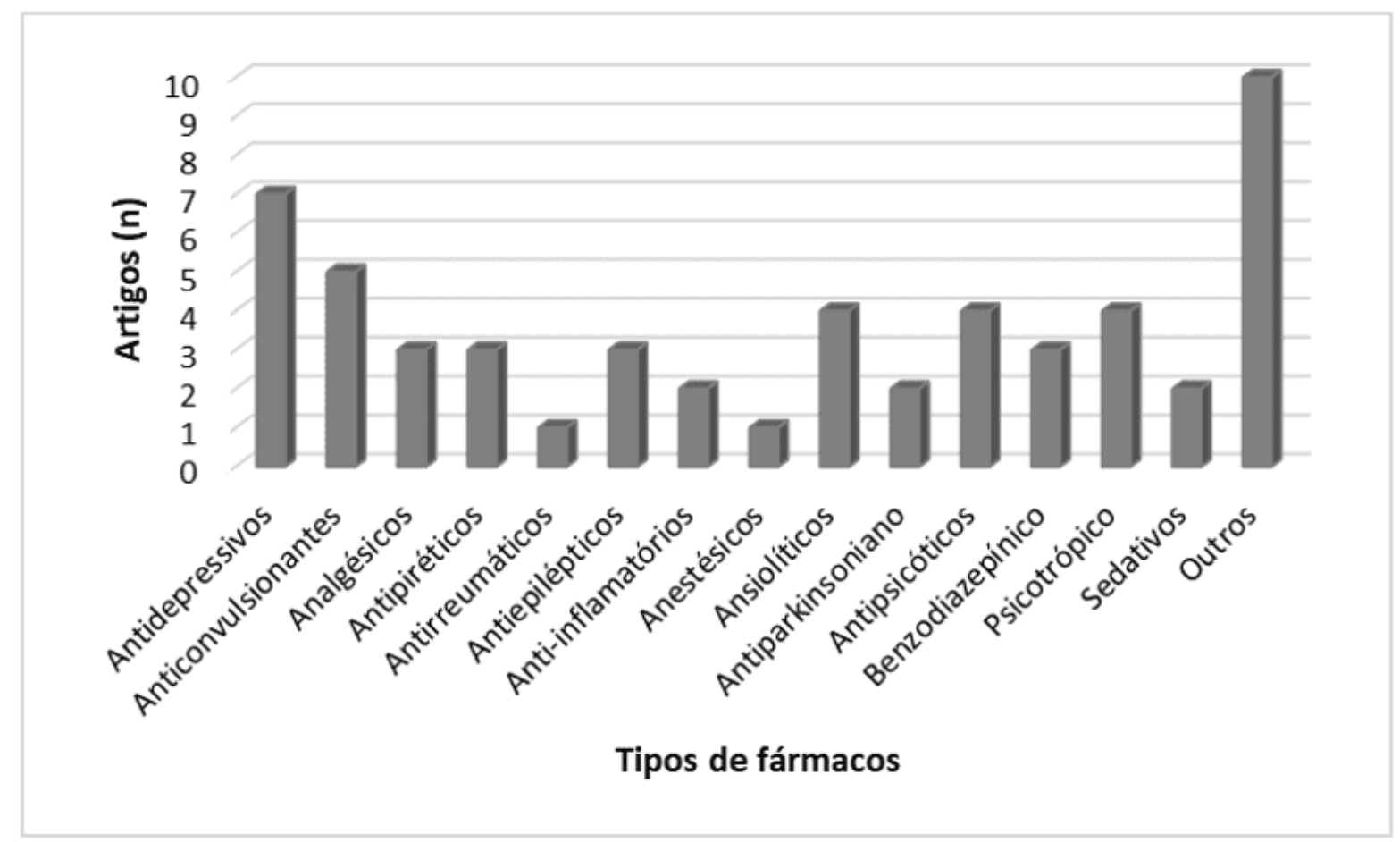

Fonte: Autor.

Segundo Taylor et al. (2014), os grupos de medicamentos partilham características similares, como o mecanismo de ação, composição química ou ação terapêutica, como é o caso dos antidepressivos que foram apontados em vários estudos como sendo a classe farmacológica mais relacionada aos casos de intoxicação por 
motivação suicida (BEREZOVSK, 2009; MAGALHÃES et al., 2013; ALEXANDRE; MARTINS, 2015; COSTA; ALONZO, 2015).

Mota et al. (2012), mostraram que os anticonvulsivantes, sedativos, antiparkinsonianos e psicotrópicos foram os tipos mais usados em intoxicações por motivação suicida. No Piauí, Magalhães et al. (2013), verificaram que os benzodiazepínicos, anticonvulsivantes, antidepressivos e neurolépticos foram os medicamentos mais utilizados, tanto de maneira isolada quanto em associação com outros fármacos. Em Barra do Garças-MT, houve predomínio do uso de medicamentos com ação no sistema nervoso central, como anestésicos, analgésicos, antiepilépticos, antiparkisonianos, psicolépticos e psicoanalépticos (OLIVEIRA; SUCHARA, 2014b). Segundo Vieira, Santana e Suchara (2015), os fármacos com ação no sistema nervoso foram os preferidos pelas vítimas, sendo responsáveis por $60,8 \%$ das intoxicações em tentativas de suicídio, com predominância no sexo feminino.

De acordo com Mota et al. (2012), o alto índice de uso de medicamentos por motivação suicida, pode ser um reflexo do padrão de consumo exagerado dos medicamentos no país. Ações de conscientização sobre o uso moderado de medicamentos são necessárias. Além de maior atenção, para aquelas pessoas que apresentam transtornos mentais, considerando que a possibilidades de estas tentarem suicídio é maior.

Observamos em $76,92 \%$ dos artigos analisados que o sexo feminino foi mais apontado como o que utiliza medicamentos com intuito de tirar a vida. Entretanto $15,38 \%$ dos artigos identificaram maior ocorrência em homens e 7,69\% não analisaram os casos no sexo masculino. Quanto a faixa etária mais atingida, citadas nos artigos, destaca-se os indivíduos com 20 a 40 anos, como sendo os que mais provocam intoxicação medicamentosa com motivação suicida.

Nesse sentido, Souza et al. (2011) observaram predominância nas tentativas de suicídio no sexo feminino, mas a consumação do ato suicida foi seis vezes maior no sexo masculino, em comparação ao sexo feminino. Esses autores verificaram que 
houve concentração dos casos de suicídio na faixa etária de 40 anos ou mais, enquanto os casos de tentativas, concentrou-se entre 20 e 39 anos.

Segundo Mota et al. (2012), entre 1996 e 2005 no Brasil, foram identificados 4.403 óbitos ocorridos em homens (53,9\%), solteiros (53,7\%) e faixa etária de 20-39 anos (44,0\%). Santos, Legay e Lovisi (2013), observaram que $82,1 \%$ das intoxicações medicamentosas com motivação suicida ocorrem entre adultos de 20 a 59 anos.

No estudo de Magalhães et al. (2013), foi verificado que $55,67 \%$ das intoxicações acometeram o sexo feminino, 59,44\% na faixa etária de 0 a 19 anos, seguida de 20 a 39 anos, com $25 \%$ dos casos. Santos et al. (2013), também mostraram que indivíduos do sexo feminino fizeram mais uso de medicamentos em casos de suicídio, comparado ao masculino, os adultos de 20 a 49 anos representaram o maior número dos casos registrados.

Um estudo com mulheres em idade fértil, expostas a medicamentos entre 2007 e 2011, constatou que maioria das ocorrências (90,5\%) foi intencional. Os medicamentos com atuação no Sistema Nervoso Central foram responsáveis por 59,9\% das ocorrências (AKAHAMA; TURINI; GIROTTO, 2014).

Ao analisar entre janeiro de 2013 e janeiro de 2014, o perfil dos atendimentos hospitalares por tentativa de suicídio em um hospital de referência da zona Norte do Estado do Ceará, compreendendo mais de 55 municípios da região, os resultados indicaram a predominância de indivíduos adolescentes e adultos jovens do sexo feminino que utilizam medicamentos como agente tóxico (OLIVEIRA et al., 2015).

Em um hospital de ensino, no interior do Rio Grande do Sul, no qual foram analisados os prontuários eletrônicos das avaliações psiquiátricas do primeiro quadrimestre do ano de 2015 , foram registradas 25 tentativas de suicídio por medicamento, dos quais se destacam os medicamentos de controle especial. Em 76\% dos casos de tentativas de suicídio eram do feminino, com média de idade encontrada foi de 34 anos (BRIXNER et al., 2016). 
Ao descrever casos de tentativa de suicídio por exposição a agentes tóxicos registrados pelo Centro de Informação e Assistência Toxicológica em Fortaleza, Ceará, Gondim et al., (2017), verificaram que os medicamentos estavam entre os agentes tóxicos mais usados nas tentativas de suicídio, em alguns casos houve utilização de dois ou mais medicamentos simultaneamente. A maioria dos casos envolvia pessoas do sexo feminino. Os indivíduos mais afetados tinham entre 12 e 39 anos, com média de idade de 21 anos.

Oliveira e Suchara (2014b) acreditam que isso se deve à idade mais produtiva do ser humano, onde o cotidiano cria pressões psicológicas, levando à utilização de medicamentos desnecessária, podendo ser resultante da automedicação, inclusive com finalidade de pôr fim à vida.

Analisando os resultados encontrados, percebemos que houve predominância do ato suicida no sexo feminino, corroborando com a maioria dos estudos que apontam maior predominância do suicídio em homens e de tentativas em mulheres. A acessibilidade aos medicamentos, principalmente por adultos pode ser determinante na escolha desse agente tóxico para uma vítima cometer ou não o ato suicida (PORDEUS et al., 2009; VIEIRA; SANTANA; SUCHARA, 2015).

As mulheres tendem a utilizar métodos menos efetivos de findar a própria vida, como a intoxicação por fármacos, enquanto os homens, apesar de fazerem menos tentativas, utilizam instrumentos e armas mais letais, sendo que estes possuem maior representatividade nos casos de suicídio propriamente dito (PORDEUS et al., 2009; MACENTE, L. B.; SANTOS, E.G.; ZANDONADE, 2009).

\section{CONSIDERAÇÕES FINAIS}

O suicídio caracteriza-se como um grave problema de saúde pública, e um desafio a ser enfrentado. $O$ uso de medicamentos como agente tóxico capaz de tirar a vida tem se tornado um meio cada vez mais comum, principalmente entre indivíduos do sexo feminino, jovens com idade entre 20 a 49 anos, que preferem métodos menos mutilantes, sendo os antidepressivos e psicotrópicos os mais utilizados. 
No Brasil, o assunto merece cuidado especial, sendo que o país está entre os dez, no ranking mundial de mortes por suicídio, uma vez que as taxas de intoxicações medicamentosa com fins suicidas tem aumentado.

Nesse sentido, é importante destacar que o suicídio, na maioria dos casos, ocorre em função de algum quadro clínico pré-existente de transtorno mental. Os casos mais comuns envolvem depressão, transtorno bipolar e esquizofrenia, entretanto casos mais simples, como problemas familiares, amorosos e profissionais também podem desencadear em tentativas, que se não evitadas pode consumar o ato do suicídio.

Embora o número de intoxicações medicamentosas autoprovocadas venha crescendo no Brasil, poucos estudos procuraram investigar os tipos de fármacos mais utilizados em tentativas de autoextermínio, havendo, portanto, a necessidade desse levantamento para a tomada de decisões estratégicas com fins preventivos. Desse modo, espera-se que o presente trabalho instigue novos estudos que investiguem os registros dos tipos farmacológicos mais utilizados em intoxicações com motivação suicida.

\section{REFERÊNCIAS}

ALEXANDRE, A. C. B.; MARTINS, H.A. Avaliação da frequência mortalidade entre adultos jovens por intoxicação medicamentosa na $14^{\underline{a}}$ regional de saúde do paraná. IX EPCC - Encontro Internacional de Produção Científica UniCesumar. Nov. 2015, n. $9, \quad$ p. $\quad 4-8 . \quad$ Disponível em: http://www.cesumar.br/prppge/pesquisa/epcc2015/anais/ana_caroline_barbosa_alex andre.pdf. Acesso em: 10/06/2018.

ASSOCIAÇÃO BRASILEIRA DE PSIQUIATRIA-ABP. Suicídio: informando para prevenir. Comissão de Estudos e Prevenção de Suicídio. Brasília: CFM/ABP, 2014. Disponível em: https:/www.cvv.org.br/wpcontent/uploads/2017/05/suicidio_informado_para_prevenir_abp_2014.pdf. Acesso em: 20/04/2018. 
BAPTISTA, M. N., MORAIS, P. R.; SISTO, F. F. (2007). Dicas para divulgação de seus trabalhos de pesquisa. Em: M. N. Baptista \& D. C. Campos (Orgs.), Metodologias de Pesquisa em Ciências: análises quantitativas e qualitativas. Rio de Janeiro: LTC.

BEREZOVSK, O. Intoxicação medicamentosa no Brasil. Brasília: Atlas médico, 2009.

BOTEGA, N. et al. Prevenção do suicídio. Debates Psiquiatria Hoje. Rio de Janeiro, a2, n1, p. 10-20, 2010.

CABALLERO-VALLES, P. J. et al. Vigilancia epidemiológica de la intoxicación aguda en el área sur de la Comunidad de Madrid: estudio VEIA 2004. An. Med. Interna (Madrid), v. 25, n. 6, p. 262-268, 2008. Disponível em: http://scielo.isciii.es/scielo.php?script=sci_arttext\&pid=S0212-71992008000600003. Acesso em: 21/04/2018.

COSTA, A. O.; ALONZO, H. G. A. Casos de Exposições e Intoxicações por medicamentos registrados em um Centro de Controle de Intoxicações do interior do Estado de São Paulo. Revista Brasileira de Pesquisa em Saúde, v. 17, p. 52-60, n. 2015. Disponível em: file://C:/Users/Cliente/Downloads/13188-34554-1-SM.pdf. Acesso em: 15/06/2018.

FEUSER, P. E. Perfil das intoxicações medicamentosas no estado de Santa Catarina. Rev. Saúde Públ. Santa Cat., v. 6, n. 2, p. 23-32, 2013. Disponível em: http://revista.saude.sc.gov.br/index.php/inicio/article/view/183. Acesso em: $30 / 05 / 2018$.

FIOCRUZ. SISTEMA NACIONAL DE INFORMAÇÕES TÓXICO FARMACOLÓGICAS- SINITOX. Dados de intoxicação. Rio de Janeiro: SINITOX/CICT/Fiocruz, 2014. Disponível em: https://sinitox.icict.fiocruz.br/. Acesso em: $21 / 04 / 2018$. 
GONDIM, ANA PAULA SOARES; NOGUEIRA, R. R.; LIMA, J. G. B.; LIMA, R. A. C.; ALBUQUERQUE, P. L. M. M.; VERAS, M. S. B.; Ferreira, M. A. D. Tentativas de suicídio por exposição a agentes tóxicos registradas em um Centro de Informação e Assistência Toxicológica em Fortaleza, Ceará, 2013. Epidemiologia e Servicos de Saude, v. 26, p. 109-119, 2017. Disponível em: http://www.scielo.br/pdf/ress/v26n1/2237-9622-ress-26-01-00109.pdf. Acesso em: 10/08/2018.

GUNNELL, D.; EDDLESTON M. Suicide by intentional ingestion of pesticides: a continuing tragedy in developing countries. Int J Epidemiol. 2003;32 (6):902-9. Disponível em: https://www.ncbi.nlm.nih.gov/pmc/articles/PMC2001280/pdf/nihms741.pdf. Acesso em: 21/04/2018.

MACENTE, L. B.; SANTOS, E.G.; ZANDONADE, E. Tentativas de suicídio e suicídio em município de cultura pomerana no interior do estado do Espírito Santo. J Bras Psiquiatr. 2009; 58(4): 238-44. Disponível em: http://www.scielo.br/pdf/jbpsiq/v58n4/a04v58n4.pdf. Acesso em: 10/08/2018.

MACHADO, D. B.; SANTOS, D. N. Suicídio no Brasil, de 2000 a 2012. Jornal Brasileiro de Psiquiatria (UFRJ. Impresso), v. 64, p. 45-54, n. 2015. Disponível em: http://www.scielo.br/pdf/jbpsiq/v64n1/0047-2085-jbpsiq-64-1-0045.pdf. Acesso em: 04/06/2018

MAGALHÃES, J. V. et al. Caracterização das intoxicações medicamentosas registradas no Centro de Informações Toxicológicas do Piauí no período de 2007 a 2012. R. pesq.: cuid. fundam (Online), v. 5, p. 55-63, n. 2013. Disponível em: https://www.redalyc.org/articulo.oa?id=505750944007. Acesso em: 10/06/2018.

MORAIS, I. C. O. et al. Perfil epidemiológico das intoxicações medicamentosas registradas pelo Centro de Assistência e Informação Toxicológica de Campina Grande (PB) no período de 2005 a 2007. Revista Brasileira de Farmácia, v.98, n.4, p.352357 , 2008.

Disponível

em: 
http://www.rbfarma.org.br/files/pag_352a357_perfil_epidemiologico.pdf. Acesso em: 22/04/2018.

MOREIRA, D. L.; MARTINS, M. C.; GUBERT, F. A.; SOUSA, F. S. P. Perfil de pacientes atendidos por tentativa de suicídio em um centro de assistência toxicológica. Ciencia y Enfermería (En línea), v. 21, p. 63-75, n. 2015. Disponivel em: https://scielo.conicyt.cl/pdf/cienf/v21n2/art_07.pdf. Acesso em: 22/0/2018.

MOTA, D. M. et al. Perfil da mortalidade por intoxicação com medicamentos no Brasil, 1996-2005: retrato de uma década. Ciênc. Saúde Coletiva, Rio de Janeiro, v. 17, n. 1, p. 61-70, 2012. Disponível em: http://www.scielo.br/pdf/csc/v17n1/a09v17n1.pdf. Acesso em: 15/06/2018.

OLIVEIRA, D. H., SUCHARA, E. A. (A). Intoxicações medicamentosas em hospital público de Barra do Garças-MT, no período de 2006 a 2009. Revista Ciências Médicas e Biológicas, v. 13, p. 55-59, n. 2014b. disponível em: https://portalseer.ufba.br/index.php/cmbio/article/view/10117/8718. Acesso em: 15/06/2018.

OLIVEIRA, E. N.; FELIX, T. A.; MENDONCA, C. B. L.; SOUZA, D. R.; FERREIRA, G. B.; FREIRE, M. A.; TEODOSIO, T. B. T.; LINHARES, J. M.; ALMEIDA, P. C. Tentativa de suicídio por intoxicação exógena: contexto de notificações compulsórias. Revista Eletrônica Gestão \& Saúde, v. 6, p. 2497-2511, 2015. Disponível em: http://periodicos.unb.br/index.php/rgs/article/view/3125/2811. Acesso em: 20/08/2018.

OLIVEIRA, F. F. S.; SUCHARA, E. A. Perfil epidemiológico das intoxicações exógenas em crianças e adolescentes em município do Mato Grosso. Revista Paulista de Pediatria (Impresso), v. 32, n. 4, p. 299-305, 2014a. disponível em: https://www.sciencedirect.com/science/article/pii/S0103058214000045. Acesso em: 30/05/2018.

PORDEUS, A.M.J.; CAVALCANTI, L.P.G.; VIEIRA, L.J.E.S.; CORIOLANO, L.S.; OSÓRIO, M.M.; PONTE, M.S.R. et al. Tentativas e óbitos por suicí- dio no município 
de Independência, Ceará, Brasil. Cien Saude Colet. 2009; 14(5): 1731-40. Disponível em: http://www.scielo.br/pdf/csc/v14n5/14.pdf. Acesso em: 20/07/2018.

SANTOS, S. A.; LEGAY, L. F.; LOVISI, G. M.; SANTOS, J. F.C.; LIMA, L.A. Suicídios e tentativas de suicídios por intoxicação exógena no Rio de Janeiro: análise dos dados dos sistemas oficiais de informação em saúde, 2006-2008*. Rev. bras. epidemiol. São Paulo , v. 16, n. 2, p. 376-387, June 2013. Disponível em: http://www.scielo.br/pdf/rbepid/v16n2/1415-790X-rbepid-16-02-00376.pdf.

Acesso em: 10/07/2018.

SANTOS, S. A.; LEGAY, L.F.; LOVISI, G. M. Substâncias tóxicas e tentativas e suicídios: considerações sobre acesso e medidas restritivas. Cad. Saúde Colet., Rio de Janeiro, v. 21, n. 1, p. 53-61, 2013. Disponível em: http://www.scielo.br/pdf/cadsc/v21n1/a09.pdf. Acesso em 10/07/2018.

SOUZA, V. S.; ALVES, M. S.; LINO, D. C. S. F.; NERY, A. A.; CASOTTI, C. A. Tentativas de suicídio e mortalidade por suicídio em um município no interior da Bahia. Jornal Brasileiro de Psiquiatria (UFRJ. Impresso), v. 60, p. 294-300, n. 2011. Disponível em: http://www.scielo.br/pdf/jbpsiq/v60n4/a10v60n4.pdf. Acesso em: 20/06/2018.

SPILLER, H.A.; APPANA, S.; BROCK, G.N. Epidemiological trends of suicide and attempted suicide by poisoning in the US: 2000-2008. Leg Med (Tokyo). 2010;12(4):177-83. Disponível em: https://www.ncbi.nlm.nih.gov/pubmed/20547089. Acesso em: 20/04/2018.

TAKAHAMA, C. H.; TURINI, C. A.; GIROTTO, E. Perfil das exposições a medicamentos por mulheres em idade reprodutiva atendidas por um Centro de Informações Toxicológicas. Ciênc. saúde coletiva, Rio de Janeiro, v. 19, n. 4, p. 1191-1199, Apr. 2014 . Disponível em: http://www.scielo.br/pdf/csc/v19n4/14138123-csc-19-04-01191.pdf. Acesso em: 10/06/2018.

TAYLOR, C.R.; LILLIS, C.; LEMONE, P.; LYNN, P. Fundamentos de Enfermagem: A Arte e a Ciência do Cuidado de Enfermagem. 7.ed. 1769 p. Artmed Editora, 2014. 
VÄRNIK, A.; KÕLVES K, ALLIK J, ARENSMAN E, AROMAA E, VAN AUDENHOVE $C$, et al. Gender issues in suicide rates, trends and methods among youths aged 1524 in 15 European countries. J Affect Disord. 2009;113(3):216-226. Disponível em: https://www.ncbi.nlm.nih.gov/pubmed/18625519. Acesso em: 22/04/2018.

VIEIRA, D. M.; CAVEIÃO, C. Perfil das intoxicações medicamentosas no Estado de São Paulo na perspectiva da vigilância sanitária. Revista Saúde e Desenvolvimento, v. 9, p. 119-141, 2016. Disponível em: file:///C:/Users/Cliente/Downloads/521-2048-1PB.pdf. Acesso em: 05/06/2018.

VIEIRA, L. P.; SANTANA, P.; SUCHARA, E. A. Caracterização de tentativas de suicídios por substâncias exógenas. Cadernos Saúde Coletiva, v. 23, p. 118-123, n. 2015. Diponivel em: http://www.scielo.br/pdf/cadsc/v23n2/1414-462X-cadsc-23-2118.pdf. Acesso em: 20/06/2018.

WAISELFISZ, J. J. Mapa da Violência: Os Jovens do Brasil. Brasília, 2014. Disponível em: https://www.mapadaviolencia.org.br/pdf2014/Mapa2014_JovensBrasil.pdf. Acesso em: 20/04/2018.

WORLD HEALTH ORGANIZATION (WHO); Krug EG et al. (ed.). World report on violence and Health. Geneva: WHO; 2002. Disponível em: http://apps.who.int/iris/bitstream/handle/10665/42495/9241545615_eng.pdf;jsessioni $d=8657 F 974$ D8670B9175680DC644522ADD?sequence=1. Acesso em: 20/04/2018.

ZHONG, B. L; CHIU, H.F.; CONWEL, Y. Rates and characteristics of elderly suicide in China. J Affect Disord. 2016; 206: 273-97. Disponível em: https://www.ncbi.nlm.nih.gov/pubmed/27639861. Acesso em: 20/04/2018.

Enviado: Janeiro, 2019.

Aprovado: Setembro, 2019. 